Yôto Kubota, Department of Mathematical Science, Ibaraki University, Mito, Ibaraki, 310, Japan

\title{
NOTES ON ABSOLUTELY CONVERGENT INTEGRATION
}

\begin{abstract}
We show that there exists an absolutely convergent integral which properly includes the Lebesgue integral and that any such integral does not have positive property as a functional.
\end{abstract}

Let $I=[a, b]$ be a fixed compact interval on $R$. We consider only real valued measurable functions defined on $I$.

Definition 1. Let $T$ be a functional operation by which there corresponds to each closed interval $J \subset I$ a linear class $K(T, J)$ of measurable functions defined on $J$, and to each function $f$ of $K(T, J)$ a real number $T(f, J)$. A functional operation $T$ is called an absolutely convergent integral if the following conditions are fulfilled:

(i) The operation $T(f, J)$ is linear on $K(T, J)$.

(ii) If $f \in K(T, J), J^{\prime} \subset J$ then $f \in K\left(T, J^{\prime}\right)$.

(iii) If $J_{1}$ and $J_{2}$ are abutting intervals and if $f \in K\left(T, J_{1}\right) \cap K\left(T, J_{2}\right)$ then $f \in K\left(T, J_{1} \cup J_{2}\right)$ and

$$
T\left(f, J_{1} \cup J_{2}\right)=T\left(f, J_{1}\right)+T\left(f, J_{2}\right) .
$$

(iv) If $f \in K(T, J), J=[c, d]$ then $F(x)=T(f,[c, x])$ is continuous on $J$.

(v) If $f \in K(T, J)$ then $|f| \in K(T, J)$.

Definition 2. Given two absolutely convergent integrals $T_{1}$ and $T_{2}$, we shall say that $T_{2}$ includes $T_{1}$ if $f \in K\left(T_{1}, J\right)$ then $f \in K\left(T_{2}, J\right)$ and $T_{1}(f, J)=$ $T_{2}(f, J)$ for any interval $J \subset I$, and write $T_{1} \subset T_{2}$.

Key Words: absolutely convergent integration, Lebesgue integral

Mathematical Reviews subject classification: Primary: 26A42, 26A30

Received by the editors September 16,1996 
The Lebesgue integral is an absolutely convergent integral. The following problem arises: Is there any absolutely convergent integral which properly includes the Lebesgue integral? We shall show that the answer to this question is "yes", however any such integral has no longer positive property as a functional.

Theorem 1. There is an absolutely convergent integral which properly includes the Lebesgue integral.

To prove the theorem, we need the following lemma.

Lemma 1. There is a measurable function $\psi: I \rightarrow(0, \infty)$ such that $(L) \int_{J} \psi$ $=+\infty$ for any subinterval $J$ of $I$.

The construction of such a function will be given later.

Proof of Theorem 1. For $J=[c, d]$, let

$$
K(T, J)=\{g: g=f+p \psi, f \in L(J), p \in R\}
$$

where $\psi$ is the function obtained in Lemma, and define

$$
T(g, J)=(L) \int_{J} f+p(d-c)
$$

Then $K(T, J)$ is a linear class and we can see easily that $T$ satisfies conditions (i) (iv). To show that if $f+p \psi \in K(T, J)$ then $|f+p \psi| \in K(T, J)$, it is sufficient to prove $|f+\psi| \in K(T, J)(f \in L(J))$.

Let $A=\{x \in J: f(x)+\psi(x) \geq 0\}$ and $B=\{x \in J: f(x)+\psi(x)<0\}$. Then the sets $A$ and $B$ are measurable. Let

$$
g(x)= \begin{cases}f(x) & (x \in A) \\ -f(x)-2 \psi(x) & (x \in B)\end{cases}
$$

We can write

$$
g(x)=f(x) \chi_{A}(x)+(-f(x)-2 \psi(x)) \chi_{B}(x),
$$

and for $x \in B, 0<\psi(x)<-f(x)$. Consequently $\psi(x) \chi_{B}(x)$ is L-integrable on $J$ and hence $g \in L(J)$. On the other hand, by the definition of $g$, we have $|f+\psi|=g+\psi$. It follows that $|f+\psi| \in K(T, J)$. Since $K(T, J)$ includes $L(J)$ strictly, the proof is complete.

Proof of Lemma 1. For simplicity, we assume $[a, b]=[0,1]$. Let $\left\{t_{k}\right\}$ be a sequence with $0<t_{k}<\frac{1}{2}$. 
We define the sequence of sets as follows:

$$
\begin{gathered}
I_{1}^{1}=\left[0, t_{1}\right], \quad I_{1}^{2}=\left[1-t_{1}, 1\right] \\
E_{1}=I_{1}^{1} \cup I_{1}^{2} \\
I_{2}^{1}=\left[0, t_{1} t_{2}\right], I_{2}^{2}=\left[t_{1}-t_{1} t_{2}, t_{1}\right], I_{2}^{3}=\left[1-t_{1}, 1-t_{1}+t_{1} t_{2}\right], I_{2}^{4}=\left[1-t_{1} t_{2}, 1\right] \\
E_{2}=I_{2}^{1} \cup I_{2}^{2} \cup I_{2}^{3} \cup I_{2}^{4}
\end{gathered}
$$

Repeating this process we can define $I_{k}^{j}$ and $E_{k}=\cup_{j=1}^{2^{k}} I_{k}^{j}$. Then the set $E=\cap_{k=1}^{\infty} E_{k}$ is perfect and

$$
m\left(I_{k}^{j}\right)=t_{1} t_{2} \cdots t_{k}, \quad m(E)=\lim _{k \rightarrow \infty} 2^{k} t_{1} t_{2} \cdots t_{k} .
$$

If we choose $t_{k}=\frac{1}{2}-\frac{1}{2(k+1)^{2}}(k=1,2, \ldots)$ then $m(E)=\frac{1}{2}$ by the formula $\Pi_{k=2}^{\infty}\left(1-\frac{1}{k^{2}}\right)=\frac{1}{2}$. Change the notations $E$ and $I_{k}^{j}$ for $E^{1}$ and $I_{1 k}^{j}$ respectively, that is,

$$
E^{1}=\cap_{k=1}^{\infty} E_{1 k}, \quad E_{1 k}=\cup_{j=1}^{2^{k}} I_{1 k}^{j} .
$$

Next we set $[0,1]-E_{1 k}=\cup_{j=1}^{2^{k-1}} I_{2 k}^{j}$, where $I_{2 k}^{j}$ are complementary closed intervals of $E_{1 k}$, and construct a perfect set $E_{2 k}^{j}$ on each $I_{2 k}^{j}$ such that $m\left(E_{2 k}^{j}\right)=$ $\frac{1}{2} \cdot m\left(I_{2 k}^{j}\right)$. Putting $E^{2}=\cup_{k} \cup_{j} E_{2 k}^{j}$, we have

$$
\begin{aligned}
m\left(E^{2}\right) & =m\left(E_{21}^{1}\right)+\sum_{k=1}^{\infty} \sum_{j=1}^{2^{k}} m\left(E_{2 k}^{2^{k}}\right) \\
& =\frac{1}{2}\left|I_{21}^{1}\right|+\sum_{k=1}^{\infty} 2^{k} \cdot \frac{1}{2}\left|I_{2 k}^{j}\right| \\
& =\frac{1}{2}\left(1-2 t_{1}\right)+\sum_{k=1}^{\infty} 2^{k-1} t_{1} t_{2} \cdots t_{k}\left(1-2 t_{k+1}\right) \\
& =\frac{1}{2^{3}}+\sum_{k=1}^{\infty} \frac{1}{2^{2}}\left(\frac{1}{k+1}-\frac{1}{k+2}\right)=\frac{1}{2^{2}} .
\end{aligned}
$$

Similarly we can define the set $E^{3}$ on $[0,1]-E^{1} \cup E^{2}$ such that $m\left(E^{3}\right)=\frac{1}{2^{3}}$ and so on. The sets $E^{n}$ thus obtained are non-overlapping and $m\left(\cup_{n=1}^{\infty} E^{n}\right)=1$, that is, $m\left([0,1]-\cup_{n=1}^{\infty} E^{n}\right)=0$.

Now we define the function $\psi:[0,1] \rightarrow(0, \infty)$ as follows:

$$
\psi(x)= \begin{cases}\frac{1}{t_{1} t_{2} \cdots t_{k}} & \left(x \in E^{k}\right), \\ 1 & \left(x \in[0,1]-\cup_{n=1}^{\infty} E^{n}\right),\end{cases}
$$


where $t_{k}=\frac{1}{2}-\frac{1}{2(k+1)^{2}}$. Since $\psi$ is positive and measurable, it remains to show that $(L) \int_{J} \psi=+\infty$ for any subinterval $J$ of $[0,1]$.

For any $J$, there is an interval $I_{n m}^{j} \subset J$. Since

$$
\begin{aligned}
\int_{I_{n m}^{j}} \psi & =\sum_{k=n}^{\infty} \int_{E^{k} \cap I_{n m}^{j}} \psi \\
& =\sum_{k=n}^{\infty} \frac{m\left(E^{k} \cap I_{n m}^{j}\right)}{t_{1} t_{2} \cdots t_{k}}>\sum_{k=n}^{\infty} \frac{1}{2^{k}}\left|I_{n m}^{j}\right| 2^{k} \\
& =\sum_{k=n}^{\infty}\left|I_{n m}^{j}\right|=+\infty,
\end{aligned}
$$

we have $\int_{J} \psi=+\infty$.

Theorem 2. If $T$ is any absolutely convergent integral which includes strictly the Lebesgue integral, then $T$ is not positive.

Proof. Suppose that if $f \in K(T, J)$ and $f \geq 0$ then $T(f, J) \geq 0$.

For any $f \in K(T, J)$ with $f \geq 0$, we set for each $n$

$$
f_{n}(x)= \begin{cases}f(x), & \text { if } 0 \leq f(x) \leq n, \\ n, & \text { if } f(x)>n\end{cases}
$$

Then $f_{n} \in L(J), f_{n} \leq f_{n+1}$ for every $n$ and $f_{n} \rightarrow f(n \rightarrow \infty)$ on $J$. Since $L(J) \subset K(T, J)$ and $T$ is positive, we have $L\left(f_{n}, J\right) \leq L\left(f_{n+1}, J\right) \leq T(f, J)$. By the monotone convergence theorem of the Lebesgue integral, we have $f \in$ $L(J)$. Thus we have showed that if $f \in K(T, J)$ and $f \geq 0$ then $f \in L(J)$.

Let $f \in K(T, J)$. Then by $(\mathrm{v}),|f| \in K(T, J)$, and hence $|f| \in L(J)$ that is, $f \in L(J)$ which implies $K(T, J) \subset L(J)$. This is a contradiction.

Acknowledgement. The greater part of this paper is due to his reports by Fu Shusheng (Professor, Fuzhou University, China). I would like to express my warmest thanks to him.

\section{References}

[1] Y. Kubota, Notes on integration, Math. Japon., 31 (1986), 617-621.

[2] Y. Kubota, A note on the Lebesgue integral, Soochow J. Math., 13 (1987), 65-67.

[3] K. Kartak, A generalization of the Carathéodory theory of the differential equations, Czech. Math. J., 17 (1967), 482-514 (especially 484-487, 510-511). 